\title{
Characterization Of a G Protein a Subunit Encoded Gene From The Dimorphic Fungus-Tremella Fuciformis
}

Hanyu Zhu

Hengyang Normal University

Dongmei Liu

Huazhong Agricultural University

Liesheng Zheng

Huazhong Agricultural University

Liguo Chen

Huazhong Agricultural University

Aimin Ma ( $\nabla$ aiminma@mail.hzau.edu.cn )

Huazhong Agricultural University https://orcid.org/0000-0003-4728-1201

\section{Research Article}

Keywords: Tremella fuciformis, dimorphism, G protein a subunit, gene function, morphological change

Posted Date: June 18th, 2021

DOl: https://doi.org/10.21203/rs.3.rs-586507/v1

License: (9) This work is licensed under a Creative Commons Attribution 4.0 International License. Read Full License 


\section{Abstract}

Tremella fuciformis is a dimorphic fungus which can undertake the reversible transition between yeast and pseudohypha forms. G protein a subunit $(\mathrm{Ga})$ carries different signals to regulate a variety of biological processes in eukaryotes, including fungal dimorphism. In this study, a novel Ga subunit encoded gene, TrGpa1, was firstly cloned from T. fuciformis. The TrGpa1 open reading frame has 1059 nucleotides, and encodes a protein which belongs to the group I of $\mathrm{Ga}_{i}$ superfamily. Furthermore, the role of TrGpa1 in the T. fuciformis dimorphism was analysed by gene overexpression and knockdown. Stable integration of the target gene into the genome was confirmed by PCR and Southern blot hybridization. Transformants with the highest and lowest TrGpa1 expression levels were selected via quantitative realtime PCR analysis and Western blot. Each transformant was compared with the wild-type strain about the morphological change under different environmental factors, including $\mathrm{pH}$ values, temperature, cultural time, inoculum size, and quorum-sensing molecules (farnesol and tyrosol). Comparing with the wild-type strain, the overexpression transformant always had higher ratios of pseudohyphae, while the knockdown transformant had less proportions of pseudohyphae. Therefore, the TrGpa1 involves in the dimorphism of T. fuciformis and plays a positive role in promoting pseudohyphal growth.

\section{Introduction}

The heterotrimeric guanine nucleotide-binding proteins (G proteins), universal signaling proteins in eukaryotes, carry different signals from the receptors to various effectors, then regulate a variety of biological processes (Kang et al. 2011). The G proteins are highly conserved, and consist of Ga, G $\beta$ and Gy subunits. In the inactive state, these three subunits are tightly associated together. Once the $\mathrm{Ga}$ subunit was activated by $\mathrm{G}$ protein-coupled receptors (GPCRs), G protein dissociates to form $\mathrm{Ga}$ and G $\beta$ Gy dimeric subunits. Each of them can interact with downstream effectors, which subsequently trigger a series of intracellular responses (Perez-Sanchez et al. 2010; Valle-Maldonado et al. 2015).

Fungal dimorphism is an intriguing morphological transition, which undertakes a morphological interconversion between the yeast form and the mycelial/pseudohypha form (Nickerson and Atkin 2017). This switch promotes the disease progression in the pathogenic filamentous species, which is necessary for the invasion to hosts and the expression of virulence factors (Wilson et al. 2010; Boyce and Andrianopoulos 2015). Lots of researches showed Ga subunits had linkage with morphological transition in dimorphic fungi. For Ustilago maydis, the yeast colonies of cap1-defective cells failed to form filamentous colonies, which resulted in a significantly decreased pathogenicity (Takach and Gold 2010). In Sporothrix schenckii, Ga subunits SSG-1 and SSG-2 involved in the dimorphism and pathogenicity (Pérez-Sánchez et al. 2010; Yemelin et al. 2017). The $\Delta M g G p a 3$ mutant of Mycosphaerella graminicola showed more pronounced yeast-like growth accompanied with hampered filamentation, which suppressed the transition from yeast-like form to filamentous form (Orton et al. 2011). As to Mucor circinelloides, the gpa3 expression levels was decreased during the dimorphic transition from mycelium to yeast cell (Patiño-Medina et al. 2019). In Candida albicans, the Gpa2 played an important role in the yeast-hypha dimorphic transition in the response of $C$. albicans to some environmental inducers 
(Wilson et al. 2010). The $\Delta g p a 2$ mutant strains of Saccharomyces cerevisiae had a defect in pseudohyphal growth, while constitutive overexpression of gpa2 stimulated filamentation of mutant (Kayikci and Magwene 2018).

Tremella fuciformis, or white jelly mushroom, is a typical dimorphic fungus having the yeast-hypha and yeast-pseudohypha transition triggered by environmental cues (Hou et al. 2011). Previous studies about the $T$. fuciformis dimorphism mainly focused on the environmental factors which affected its dimorphism and cell wall polysaccharides changes during dimorphic transition (Zhu et al. 2016). Little is known about the functions of signaling proteins during the dimorphic change of T. fuciformis. In the present study, a Ga subunit gene (TrGpa1) was cloned and characterized its contributions to the $T$. fuciformis dimorphism. The gene overexpression and knockdown vectors were constructed to evaluate its roles in the dimorphic transition in response to the environmental inducers.

\section{Materials And Methods}

\section{Strains and Culture Conditions}

The T. fuciformis haploid yeast-like cell Y32, was maintained in the Laboratory of Food Microbiology, Huazhong Agricultural University, and subcultured on potato dextrose agar (Difco, Detroit, MI, USA) slants. The $\mathrm{LM}$ medium $\left(20 \mathrm{~g} \cdot \mathrm{L}^{-1}\right.$ glucose, $1.32 \mathrm{~g} \cdot \mathrm{L}^{-1}\left(\mathrm{NH}_{4}\right)_{2} \mathrm{SO}_{4}, 0.25 \mathrm{~g} \cdot \mathrm{L}^{-1} \mathrm{MgSO}_{4} \cdot 7 \mathrm{H}_{2} \mathrm{O}, 0.5 \mathrm{~g} \cdot \mathrm{L}^{-1}$ $\mathrm{KH}_{2} \mathrm{PO}_{4} \cdot 3 \mathrm{H}_{2} \mathrm{O}, 0.2 \mathrm{mg} \cdot \mathrm{L}^{-1}$ vitamin $\mathrm{B}_{1}, 2 \mathrm{mg} \cdot \mathrm{L}^{-1} \mathrm{ZnSO}_{4} \cdot 7 \mathrm{H}_{2} \mathrm{O}$, and $0.5 \mathrm{~g} \cdot \mathrm{L}^{-1} \mathrm{CaCl}_{2} \cdot 2 \mathrm{H}_{2} \mathrm{O}$ ) was designed for strain culturing. The strains were incubated at $25^{\circ} \mathrm{C}$ using an orbital shaker (Fuma, Shanghai, China) at $150 \mathrm{rpm}$. Escherichia coli DH5a (Takara, Dalian, China) was used as a host for vectors' cloning and propagation. The Agrobacrerium tumefaciens strain EHA105 (Invitrogen, Shanghai, China), grown in YEB medium (Sigma-Aldrich, Shanghai, China) with the selective antibiotics (50 $\mu \mathrm{g} \cdot \mathrm{mL}^{-1}$ rifampicin and 100 $\mu \mathrm{g} \cdot \mathrm{mL}^{-1}$ kanamycin), was used to transform $\mathrm{Y} 32$. The T. fuciformis transformants were selected by PDSA (PDA containing $50 \mu \mathrm{g} \cdot \mathrm{mL}^{-1}$ hygromycin and $200 \mu \mathrm{g} \cdot \mathrm{mL}^{-1}$ cefotaxime sodium).

Full-length Gene Cloning and Bioinformatical Analysis

Ga subunit gene, named as TrGpa1, and the DNA (GenBank accession no. MH091706) and cDNA (GenBank accession no. MH101517) were acquired in our previous work. Total RNA and DNA were extracted from $\mathrm{Y} 32$ strain using the RNaiso ${ }^{\mathrm{TM}}$ plus (Takara, Dalian, China) and the cetyltrimethylammonium bromide (CTAB) method (Yin et al. 2015), respectively. The TrGpa 1 was cloned by PCR with the specific primers listed on Table 1. The amplification procedures were carried out as follows: an initial denaturation at $94^{\circ} \mathrm{C}$ for $5 \mathrm{~min} ; 35$ cycles of $94^{\circ} \mathrm{C}$ denaturation for $30 \mathrm{~s}, 60^{\circ} \mathrm{C}$ annealing for $30 \mathrm{~s}, 72^{\circ} \mathrm{C}$ elongation for $90 \mathrm{~s}$; and a final extension at $72^{\circ} \mathrm{C}$ for $10 \mathrm{~min}$.

Table 1 Primers for PCR amplification in this work. 


\begin{tabular}{|c|c|c|}
\hline Names & Sequences $\left(5^{\prime} \rightarrow 3^{\prime}\right)$ & Descriptions \\
\hline $\begin{array}{l}\text { TrGpa1- } \\
\text { F }\end{array}$ & CATGGGGTGCACACAGTCG & \multirow[t]{2}{*}{ Primers for TrGpa1 } \\
\hline $\begin{array}{l}\text { TrGpa1- } \\
\text { R }\end{array}$ & TCAAAGCAATCCGACCTCCC & \\
\hline $\mathrm{OE}-\mathrm{F}$ & CCTAGGATGGGGTGCACACAG & \multirow[t]{2}{*}{ Primers for TrGpa 1 overexpression } \\
\hline OE-R & TTCGAATCAAAGCAATCCG & \\
\hline $\mathrm{F}-\mathrm{F}$ & ACGCGTGGAACGAGATCAAGATGCTC & \multirow[t]{2}{*}{ Amplify a sense fragment for the RNAi vector } \\
\hline F-R & CCTAGGCGTGCACGGAGGATGTCTTGG & \\
\hline $\mathrm{R}-\mathrm{F}$ & CCTAGGGGACCGCCGGGTCCTGCCAC & \multirow{2}{*}{$\begin{array}{l}\text { Amplify an antisense fragment for the RNAi } \\
\text { vector }\end{array}$} \\
\hline $\mathrm{R}-\mathrm{R}$ & TTCGAAGGAACGAGATCAAGATGCTC & \\
\hline $\mathrm{EH}-\mathrm{F}$ & GCAGAAGAACGGCATCAAGGTG & \multirow[t]{2}{*}{ Detects the eGFP and hygromycin expression } \\
\hline EH-R & CAGGCTCTCGCTAAACTCCCC & \\
\hline qGpa1-F & CCGCCTTGGTCTTCCTCATT & \multirow[t]{2}{*}{ TrGpa 1 primers for qRT-PCR } \\
\hline qGpa1-R & TAGTTGCTCCCGCCCTTGTA & \\
\hline tubulin-F & GATGACCATTTCTTGCTTC & \multirow[t]{2}{*}{ Tubulin primers for qRT-PCR } \\
\hline $\begin{array}{l}\text { tubulin- } \\
\mathrm{R}\end{array}$ & GTTCTGACATTTGCTACCG & \\
\hline M13-F & CGCCAGGGTTTTCCCAGTCACGAC & \multirow[t]{2}{*}{ Primers for sequencing } \\
\hline M13-R & AGCGGATAACAATTTCACACAGGA & \\
\hline
\end{tabular}

The recognition sequences for restriction enzyme are underlined.

Bioinformation analysis of TrGpa1 was performed by following steps. The amino acid sequence was deduced by Translate tool (http://web.expasy.org/translate/). The theoretical isoelectric point and molecular weight were predicted using Compute pl/Mw (http://expasy.org/tools/protparam.html). The conserved domains were identified from the National Center for Biotechnology Information (NCBI) (http://www.ncbi.nlm.nih.gov/Structure/cdd/wrpsb.cgi). Sequence similarity was analysed using Basic Local Alignment Search Tool (BLAST) at the NCBI (https://blast.ncbi.nlm.nih.gov/Blast.cgi). Multisequence alignment was generated using ClustalW (http://www.clustal.org/). The phylogenetic tree was constructed using neighbor joining method implemented in Molecular Evolutionary Genetics Analysis (MEGA) version 6 program.

Vector Construction and Agrobacterium-mediated Transformation 
The overexpression and knockdown vectors of TrGpa 1 were constructed according to the vector pGEHGH (Zhu et al. 2017) based on pCAMBIA 1302 backbone (Cambia, Brisbane, Australia). The TrGpa1 amplified using the primers with Mlul and Asull restriction sites were digested and introduced into pGEH$\mathrm{GH}$ to generate the overexpression vector $\mathrm{PTrGpa1-OE} \mathrm{(Fig.} \mathrm{1a).} \mathrm{The} \mathrm{knockdown} \mathrm{vector} \mathrm{(pTrGpa1-OE)} \mathrm{was}$ generated by the ligation of a $439 \mathrm{bp}$ fragment (flanking M/ul and BInl restriction sites) and a $325 \mathrm{bp}$ (flanking BInl and Asull restriction sites) fragments. The plasmid was expected to encode a hairpin RNA included two 325 bp complementary regions separated by a 124 bp spacer fragment (Fig. 1b).

All vectors were transformed into the $A$. tumefaciens strain EHA105 component cells. Agrobacteriummediated transformation of $T$. fuciformis Y32 cells were performed according to previous work (Zhu et al. 2017). The transformants were subcultured for five rounds on PDSA, then the total DNA were extracted. The existence of enhanced green fluorescent protein gene (egfp)-hygromycin B phosphotransferase gene ( $h p h$ ) fusion gene was assessed by PCR using primers EH-F and EH-R. The integration of genes in the genome was analysed by Southern blot with digoxigenin (Roche Diagnostics, Mannheim, Germany) labeled $h p h$. DNA of Y32 was used as the negative control, and plasmid of pGEH-GH was applied as the positive control. Transformants were measured by using a fluorescence microscope (DM 6000 B, Leica Microsystems, Germany) to analyze the egfp expression. The images were captured under $40 \times$ objective and samples were measured with a green fluorescence filter $(546 \mathrm{~nm})$.

Gene Expression Analysis

Total RNA was extracted and reverse transcribed (TransScript ${ }^{\circledR}$ first-strand cDNA synthesis supermix, Transgen, China) according to the manufacturer's protocol. Quantitative real-time PCR (qRT-PCR) was performed on the ABI ViiA7 Real-Time PCR System (Applied Biosystems, USA) according to the manufacturer's protocol (Takara), using $\beta$-tubulin as the endogenous control. The primer sequences for the qRT-PCR of TrGpa 1 and $\beta$-tubulin are listed on Table 1. The qRT-PCR conditions were $95^{\circ} \mathrm{C}$ for $10 \mathrm{~min}$, followed by 30 cycles of $95^{\circ} \mathrm{C}$ for $30 \mathrm{~s}$, and $60^{\circ} \mathrm{C}$ for $30 \mathrm{~s}$. The expression ratios were calculated according to the $2^{-\triangle \triangle \mathrm{Ct}}$ method and each qRT-PCR reaction was carried out in triplicate independently. Data were analysed by one-way analysis of variance (ANOVA), followed by Ducan's multiple range tests using SPSS 26.0 software.

Transformants selected based on the qRT-PCR assays and Y32 were performed with Western blots. Cells were lysed in buffer containing protease and phosphatase inhibitor cocktails (Sigma, St. Louis, MO, USA). Then total protein content was measured with BCA Protein Assay Kit according to the manufacturer's protocol, and $40 \mu \mathrm{g}$ of proteins were separated on $10 \%$ SDS polyacrylamide gels and transferred to PVDF membrane (Thermo Fisher Scientific, USA) using a Semi Dry Blotter (Thermo Fisher Scientific, USA) for 1 $\mathrm{h}$ at 20 volts. After blocking with TBST buffer ( $5 \%$ dry milk powder in tris-buffered saline and Tween 20 ) for $1 \mathrm{~h}$, the membrane was incubated with the primary rabbit antibodies against $\operatorname{TrGpa} 1$ at $4^{\circ} \mathrm{C}$ overnight. After 5 washing steps with TBST buffer, blots were incubated with the secondary antibodies: anti-rabbit IgG in the dilution of 1:2000 in 5\% milk/TBST at room temperature for $2 \mathrm{~h}$. The membranes were performed using the SuperSignal ECL Solution for Western blot (Willget Biotech, Shanghai, China). 
Densitometric evaluation was performed with ImageJ software (National Institute of Health, New York, NY).

\section{Phenotypic Analysis}

The sub-cultured cells in LM medium were aseptically collected by centrifugation at $5000 \times g$ for 5 min, washed three times and distributed in $50 \mathrm{~mL}$ of medium to obtain final concentration of $10^{5} \mathrm{cells} \cdot \mathrm{mL}^{-1}$. Except the given situations, the $50 \mathrm{~mL}$ of LM medium containing cells were incubated at $25^{\circ} \mathrm{C}$ on an orbital shaker at $\mathrm{pH} 7$ for 3 to $5 \mathrm{~d}$. The $\mathrm{pH}$ of the medium was adjusted by the addition of dibasic phosphate-citric acid buffer to the desired $\mathrm{pH}$ value.

The transformants and $\mathrm{Y} 32$ were cultured under different conditions to test the morphological changes in Y32 and transformants. $20^{\circ} \mathrm{C}, 25^{\circ} \mathrm{C}, 28^{\circ} \mathrm{C}, 30^{\circ} \mathrm{C}, 37^{\circ} \mathrm{C}$ were chosen as the temperature parameters and 3 , $4,5,6,7,8$ as the $\mathrm{pH}$ parameters. The cells were incubated for 2 to $9 \mathrm{~d}$ for the culture time parameters. Each LM medium containing different concentrations of cells $\left(10^{3}, 10^{4}, 10^{5}, 10^{6}\right.$, and $10^{7}$ cells $\left.\mathrm{mL}^{-1}\right)$ was cultured respectively. Quorum sensing molecules (QSMs) including farnesol and tyrosol (Sigma, USA) in different concentrations $\left(5,25,50,100,200 \mu \mathrm{mol} \cdot \mathrm{L}^{-1}\right)$ were also prepared to observe their effects on morphological changes. Strains supplemented with $1 \%$ methanol were the controls for each assay. All experiments were performed in triplicate of each treatment. Data were analysed by ANOVA, followed by Ducan's multiple range tests.

Samples were observed at $20 \times$ objective by an optical microscope (Leica, Germany). Three or more cells connected at the end of the long axes or in a definite direction or an elongated cell with a daughter cell and an ellipsoidal cell having two branched daughter cells were counted as a pseudohypha. Only differentiated cells were quantified and normalized to 100\% (yeast/pseudohypha cells). For each repetition, at least 300 cells were counted under the microscopy.

\section{Results}

The Bioinformation Analysis of TrGpa1

The DNA sequence of TrGpa 1 is 1436 bp and contains eight introns of $53,61,41,40,45,47,46$, and 44 nucleotides, respectively (data not shown). The $5^{\prime}$ and 3 ' borders of the eight introns showed the same splicing sites (GT-AG) which are the common sequences for introns of filamentous fungi (Yin et al. 2015). The CDs encoded a protein of 352 amino acid residues. The calculated theoretical isoelectric point of TrGpa1 was 5.55, and the molecular weight was $40.18 \mathrm{kDa}$. The conserved domain analysis of the amino acid sequence revealed that TrGpa1 contained the GTPase domain (G1-G5), an ATP/GTP binding regions (G/AXXXXGKT/S), and the ßy complex interaction site.

Multiple sequence alignment of the predicted amino acid sequence of TrGpa1 with eight known fungal Ga subunits available in NCBI database was performed by ClustalW program. The results revealed that TrGpa1 had highly identities with other homologues from Kwoniella heveanensis (89\%, OCF36875.1), 
Cryptococcus gattii (86\%, XP_003191999.1), C. neoformans (86\%, XP_566528.1), Pisolithus sp. (66\%, AAK15759.1), Schizophyllum commune (66\%, XP_003029155.1), Laccaria bicolor (65\%, XP_001888946.1), Lentinula edodes (65\%, AAP13579.1), Hypsizygus marmoreus (65\%, KYQ31721.1) (Fig. 2).

A phylogenetic tree was constructed based on the multiple sequence alignments with the Ga subunits from other species (Fig. 3). The Ga proteins were divided into four groups according to their evolutionary relationships. It revealed that $\operatorname{TrGpa} 1$ belongs to the group I of mammalian $\mathrm{Ga}_{i}$ superfamily. The amino acid sequence of TrGpa1 contained the consensus myristoylation site, indicated as MGXXXS at the $\mathrm{N}$ terminus, but did not present a consensus CXXX sequence (pertussis toxin-catalyzed ADP-ribosylation site) at the $\mathrm{C}$ terminus (Fig. 2).

Stability Test of Transformants

Single colonies of $T$. fuciformis transformants were selected randomly and sub-cultured in PDSA. To confirm the integration of the overexpression and knockdown fragments, the DNA was extracted from 12 randomly selected transformants, and Y32. Amplification of a 500 bp DNA product, suggesting that the egfp-hph fusion gene had been transferred into these transformants (Fig. 4a). Southern blot analysis performed in 10 PCR positive transformants showed that all transformants except one appeared to have copies of the $h p h$ gene at random sites, but $Y 32$ showed no hybridization (Fig. $4 \mathrm{~b}$ ). The transformants were inspected by fluorescence microscopy, respectively. It showed stable and highly efficient eGFP expression in the individual transformants (Fig. 5).

Gene Expression Analysis of Transformants

The qRT-PCR assays were used to test the expression of TrGpa1. For the overexpression transformants (Fig. 6a), the expression level increased from 1.5 to 2.5 folds, and the transformant with the highest relative mRNA level was selected and sub-cultured. In addition, the gene suppression ratio ranged from $52.10 \%$ to $68.26 \%$ among the knockdown transformants (Fig. 6a). Thus, the transformant having the highest gene suppression ratio was chosen. Then Western blot was subsequently undertaken in Y32 and transformants with the maximum and minimum expression level. As shown in Fig 6b, TrGpa1 in the overexpression transformants was expressed at a higher level than $Y 32$, whereas at a lower level in the knockdown transformants, which is in accordance with the qRT-PCR analysis.

TrGpa1 Contributes to T. fuciformis Dimorphism

The dimorphic-related functions of TrGpa1 were characterized. It was shown that the TrGpa1 was required for pseudohyphal differentiation of $T$. fuciformis. When the environmental conditions change, the transition from yeast to pseudohypha in Y32, the overexpression and knockdown transformants were influenced by the environmental factors, including $\mathrm{pH}$, temperature, inoculum size, culture time, farnesol and tyrosol concentration (Fig. 7). Comparing with Y32, the overexpression transformant always had 
higher ratios of pseudohyphae, and the knockdown transformant always had less proportions of pseudohyphae (Fig. 7).

\section{Discussion}

The dimorphism is a reversible transition and depends upon the environment to which the fungi are exposed (Wang et al. 2020). T. fuciformis, an edible jelly mushroom, has the capacity to perform this type of morphogenesis (Zhu et al. 2016). It has been demonstrated that $\mathrm{G}$ proteins are key regulators of this morphological transition in many dimorphic fungi (Park et al. 2020). However, G proteins and their functions that are involved in T. fuciformis have been little known.

In this work, we have cloned the TrGpa1, a gene encoded Ga subunit from T. fuciformis. Filamentous fungi usually have three Ga-encoded genes that belong to three groups. According to the phylogenetic tree, $\operatorname{TrGpa1}$, the $\operatorname{TrGpa} 1$ expressed protein, belongs to the group I of $\mathrm{Ga}_{i}$ superfamily containing the characteristic sequence sites (Fig. 3). The site for myristoylation at the $\mathrm{N}$ terminus is important to attachment to the GPCRs in membrane (Li et al. 2019). The amino acid sequence of TrGpa1 did not contain the conserved pertussis toxin site at the $C$ terminus. ADP ribosylation of the $\mathrm{Ga}_{i}$ subunits locks the activity of $\mathrm{G}$ proteins and prevents the activation by GPCRs (Appleton et al. 2014).

Furthermore, the overexpression and knockdown vectors were constructed for identifying the function of TrGpa1. Since the gene knockout methods are lacking in T. fuciformis, RNA interference (RNAi) was performed for identifying the function of TrGpa1 (Wang et al. 2017). Though it causes only partial gene silencing, RNAi technology provides variable rates of gene suppression transformants. Therefore, it makes the possibility to investigate the effects of genes on the phenotypes of interest and the minimum effective inhibition rate. For example, in Magnaporthe oryzae, only a slight decrease in the expression of some calcium signaling related genes caused a complete loss of infection-related morphogenesis and pathogenicity (Lange and Peiter 2020). Nevertheless, strong knockdown of hydrophobin gene Mpg1 did not severely affect its pathogenicity, despite knockout of this gene presented a drastic reduction in pathogenicity (Han 2018). Thus, the impacts on the phenotypes would differ among different genes. Here we only chose the transformant with the highest or lowest gene expression. It is worthy of discussion how the rates of gene suppression contribute to the dimorphic phenotypes in our future work.

The characterization of dimorphic related functions indicated that TrGpa1 played a positive role in the promotion of pseudohyphal growth. The overexpression of TrGpa 1 enhances the response to different conditions and promotes the pseudohyphal formation. Cells lacking TrGpa1 have a defect in pseudohyphal development in response to specific environmental cues. For dimorphic fungi, cell morphology is depending on the inoculation size. There is a general phenomenon for all dimorphic fungi (Wedge et al. 2016), that is when inoculation at $\geq 10^{6} \mathrm{cells} \cdot \mathrm{mL}^{-1}$, budding yeasts are produced, while pseudohyphae and mycelia are produced following inoculation at $\otimes 10^{6}$ cells $\cdot \mathrm{mL}^{-1}$. In the present study, interestingly, the overexpression transformant had high ratios of pseudohyphae, even when the inoculum size was larger than $10^{6}$ cells $\mathrm{mL}^{-1}$ (Fig. 7c). In addition to the inoculation size, extracellular 
QSMs contribute to T. fuciformis dimorphism. In C. albicans, farnesol is a characterized QSM, which suppresses filamentous formation. Tyrosol, another QSM produced by C. albicans, stimulates the yeastto-hypha conversion (Han et al. 2011; Monteiro et al. 2017). In Y32 and the transformants, farnesol and tyrosol played the same roles. Farnesol effectively blocked the transition from yeast to pseudohypha at the concentration of $50 \mu \mathrm{mol} \cdot \mathrm{L}^{-1}$ (Fig. 7e), while tyrosol stimulated this transition (Fig.

7f). The pseudohyphae ratios of knockdown transformant were largely decreased in the addition of farnesol, while the pseudohyphae ratios of overexpression transformant were slightly decreased, which shows the overexpression transformant was less sensitive to the QSMs (Fig. 7).

However, the TrGpa1 involved signal pathways still remains a mystery. Former studies showed many Ga subunits are involved in the cAMP-protein kinase A (PKA) and the mitogen-activated protein kinase (MAPK) pathways (Nogueira et al. 2015; Shwab et al. 2017; Martínez-Soto et al. 2020). Here the exogenous addition of CAMP $\left(10 \mathrm{mmol} \cdot \mathrm{L}^{-1}\right)$ had no apparent influences on the yeast-pseudohypha transition, indicates TrGpa 1 may not involve in the cAMP/PKA pathway (data not shown).

In conclusion, a G protein a subunit encoded gene TrGpa 1, was cloned from the dimorphic fungus $T$. fuciformis. The TrGpa 1 encoded a protein of 352 amino acid residues and the TrGpa1 belongs to the group I of $\mathrm{Ga}_{\mathrm{i}}$ superfamily. The function of $\operatorname{TrGpa} 1$ was characterized by gene overexpression and knockdown. The results have demonstrated that TrGpa 1 involved in T. fuciformis dimorphism and supports a positive role in the transition from yeast to pseudohyphal growth under different environmental conditions. In our future study, the pathways TrGpa1 involved in and rates of gene suppression need to be further investigated.

\section{Declarations}

Author Contributions H.Z. and A.M. designed the study; L.Z. and L.C. collected the samples; H.Z. and D.L. performed the laboratory work; H.Z. performed the data analysis and wrote the manuscript; A.M., L.Z., and L.C. reviewed and revised the writing.

Funding This work was supported by grants from the National Natural Science Foundation of China (NSFC) (No. 30972072 and No. 31572182) to Aimin Ma and from the General project of Hunan Provincial Education Department (No. 19C0289) to Hanyu Zhu.

Availability of data and materials The DNA and CDNA sequences of TrGpa 1 can be downloaded from the National Center for Biotechnology Information (NCBI), and the GenBank accession numbers are $\mathrm{MH} 091706$ and $\mathrm{MH} 101517$.

\section{Compliance with ethical standards}

Conflicts of Interest The authors declare no conflicts of interest. 
Ethical statement This article does not contain any studies with human participants or animals performed by any of the authors.

Consent for publication All authors read and approved the final version of the manuscript.

\section{References}

1. Appleton KM, Bigham KJ, Lindsey CC, Hazard S, Lirjoni J, et al (2014) Development of inhibitors of heterotrimeric $\mathrm{Ga}_{i}$ subunits. Bioorg Med Chem 22:3423-3434

2. Boyce KJ, Andrianopoulos A (2015) Fungal dimorphism: the switch from hyphae to yeast is a specialized morphogenetic adaptation allowing colonization of a host. FEMS Microbiol Rev 39:797811

3. Han H (2018) RNA Interference to knock down gene expression. Methods Mol Biol 1706:293-302

4. Han TL, Cannon RD, Villasbôas SG (2011) The metabolic basis of Candida albicans morphogenesis and quorum sensing. Fungal Genet Biol 48:747-763

5. Hou LH, Chen Y, Ma CJ, Liu J, Chen LG et al (2011) Effects of environmental factors on dimorphic transition of the jelly mushroom Tremella fuciformis. Cryptogam Mycol 32:421-428

6. Kang GJ, Gong ZJ, Cheng JA, Zhu ZR, Mao CG (2011) Cloning and expression analysis of a G-protein a subunit- $\mathrm{Ga}_{\mathrm{o}}$ in the rice water weevil Lissorhoptrus oryzophilus Kuschel. Arch Insect Biochem Physiol 76:43-54

7. Kayikci Ö, Magwene PM (2018) Divergent roles for CAMP-PKA signaling in the regulation of filamentous growth in Saccharomyces cerevisiae and Saccharomyces bayanus. G3 (Bethesda) 8:3529-3538

8. Lange M, Peiter E (2020) Calcium transport proteins in fungi: the phylogenetic diversity of their relevance for growth, virulence, and stress resistance. Front Microbiol 10:3100

9. Li X, Zhong KL, Yin ZY, Hu JX, Wang WH, et al (2019) The seven transmembrane domain protein MoRgs7 functions in surface perception and undergoes coronin MoCrn1-dependent endocytosis in complex with Ga subunit MoMagA to promote cAMP signaling and appressorium formation in Magnaporthe oryzae. PLoS Pathog 15:e1007382

10. Martínez-Soto D, Ortiz-Castellanos L, Robledo-Briones M, León-Ramírez CG (2020) Molecular mechanisms involved in the multicellular growth of Ustilaginomycetes. Microorganisms 8:1072

11. Nickerson KW, Atkin AL (2017) Deciphering fungal dimorphism: farnesol's unanswered questions. Mol Microbiol 103:567-575

12. Monteiro DR, Arias LS, Fernandes RA, Deszo da Silva LF, de Castilho MOVF et al (2017) Antifungal activity of tyrosol and farnesol used in combination against Candida species in the planktonic state or forming biofilms. J Appl Microbiol 123:392-400

13. Nogueira KM, Costa MN, de Paula RG, Mendonça-Natividade FC, Ricci-Azevedo R et al (2015) Evidence of cAMP involvement in cellobiohydrolase expression and secretion by Trichoderma reesei 
in presence of the inducer sophorose. BMC Microbiol 15:195

14. Orton ES, Deller S, Brown JK (2011) Mycosphaerella graminicola: from genomics to disease control. Mol Plant Pathol 12:413-424

15. Park HS, Kim MJ, Yu JH, Shin KS (2020) Heterotrimeric G-protein signalers and RGSs in Aspergillus fumigatus. Pathogens 9:902

16. Patiño-Medina JA, Reyes-Mares NY, Valle-Maldonado MI, Jácome-Galarza IE, Pérez-Arques C et al (2019) Heterotrimeric G-alpha subunits Gpa11 and Gpa12 define a transduction pathway that control spore size and virulence in Mucor circinelloides. PLoS One 14:e0226682

17. Pérez-Sánchez L, González E, Colón-Lorenzo EE, González-Velázquez W, González-Méndez R et al (2010) Interaction of the heterotrimeric G protein alpha subunit SSG-1 of Sporothrix schenckii with proteins related to stress response and fungal pathogenicity using a yeast two-hybrid assay. BMC Microbiol 19:262

18. Shwab EK, Juvvadi PR, Waitt G, Soderblom EJ, Moseley MA et al (2017) Novel phosphoregulatory switch controls the activity and function of the major catalytic subunit of protein kinase $A$ in Aspergillus fumigatus. mBio 8:e02319-02316

19. Takach JE, Gold SE (2010) Identification and characterization of Cap1, the adenylate cyclaseassociated protein (CAP) ortholog in Ustilago maydis. Physiol Mol Plant Pathol 75:30-37

20. Valle-Maldonado MI, Jácome-Galarza IE, Díaz-Pérez AL, Martínez-Cadena G, Campos-García J et al (2015) Phylogenetic analysis of fungal heterotrimeric $\mathrm{G}$ protein-encoding genes and their expression during dimorphism in Mucor circinelloides. Fungal Biol 119:1179-1193

21. Wang S, Chen H, Tang X, Zhang H, Chen W et al (2017) Molecular tools for gene manipulation in filamentous fungi. Appl Microbiol Biotechnol 101:8063-8075

22. Wang YY, Wei XL, Bian ZY, Wei JC, Xu JR (2020) Coregulation of dimorphism and symbiosis by cyclic AMP signaling in the lichenized fungus Umbilicaria muhlenbergii. Proc Natl Acad Sci USA 117:23847-23858

23. Wedge MÈ, Naruzawa ES, Nigg M, Bernier L (2016) Diversity in yeast-mycelium dimorphism response of the Dutch elm disease pathogens: the inoculum size effect. Can J Microbiol 62:525-529

24. Wilson D, Fiori A, Brucker KD, Dijck PV, Stateva L (2010) Candida albicans Pde1p and Gpa2p comprise a regulatory module mediating agonist-induced cAMP signaling and environmental adaptation. Fungal Genet Biol 47:742-752

25. Yemelin A, Brauchler A, Jacob S, Laufer J, Heck L et al (2017) Identification of factors involved in dimorphism and pathogenicity of Zymoseptoria tritici. PLoS One 12:e0183065

26. Yin CM, Zheng LS, Zhu JH, Chen LG, Ma AM (2015) Enhancing stress tolerance by overexpression of a methionine sulfoxide reductase A (MsrA) gene in Pleurotus ostreatus. Appl Microbiol Biotechnol 99:3115-3126

27. Zhu HY, Liu DM, Wang YY, Ren DF, Zheng LS et al (2017) Use of the yeast-like cells of Tremella fuciformis as a cell factory to produce a Pleurotus ostreatus hydrophobin. Biotechnol Lett 39:11671173 
28. Zhu HY, Yuan Y, Liu J, Zheng LS, Chen LG et al (2016) Comparing the sugar profiles and primary structures of alkali-extracted water-soluble polysaccharides in cell wall between the yeast and mycelial phases from Tremella fuciformis. J Microbiol 54:381-386

\section{Figures}

a

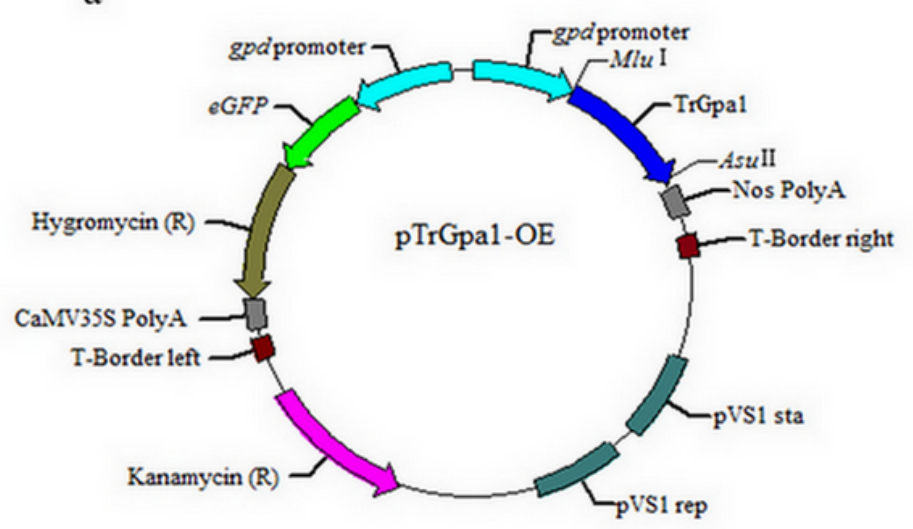

b

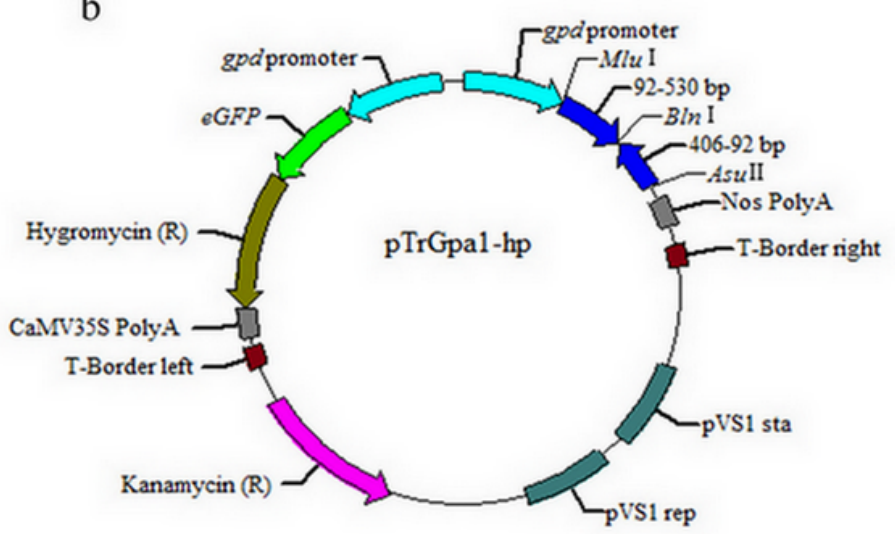

\section{Figure 1}

Map of the TrGpa1 over-expression vector (a) and knockdown vector (b). 


\author{
Aspergillus clavatus \\ Cryptococcus gattii \\ Cryptococcus neoformans \\ Laccaria bicolor \\ Lentinula edodes \\ Pisolithus sp. \\ Schizophyllum commune \\ Tremella fuciformis \\ Ustilago mavelis
}

NGCGNST. EDKEGKARNEEI TINQLKRDKNN ORNEI/KNLLLGAGESGKSTIILKQNKIIHEG

NGCTOS T. EDVAAKARS ADI DEQLKRDR ANL RNEI KNLLLGAGES GKS TVLKQNRII INK NGCTOSI. EDAAAKARS AEI DEQLKRDR ANL RNEI KNLLLGAGES GKS TVLKQNRLI YNK GCVOS S GVDDEAKARNDEI ES QLKRDRNMAKNEI KNLLL GAGES GKS TVLKQNKLI HHG NGCAOSS GI DDEAKARNDEI ENQLRRDRNNAKNEI KNLLLGAGES GKS TVLKQNKLI HHG NGCVOS IGVDGDAKARNDEI ENQLRRDKNLAKNEI KNLLLGAGES GKS IVLKQNKLI HHG GCTOS I. EDAAAKARNAEI EEQLKRDRANL RNEI KNLLLGAGES GKS TVLKQNRLI FNK AGCGASK. VDKEGQARNDAI DAQLKKDRLAQKNEI KNLLLGAGES GKS IIIKQNKLI NHG GCVOS S GVDEEAKARNDEI ES QLKKDRNAAKNEI KNLLLGAGES GKS TVLKQNKLI HHG
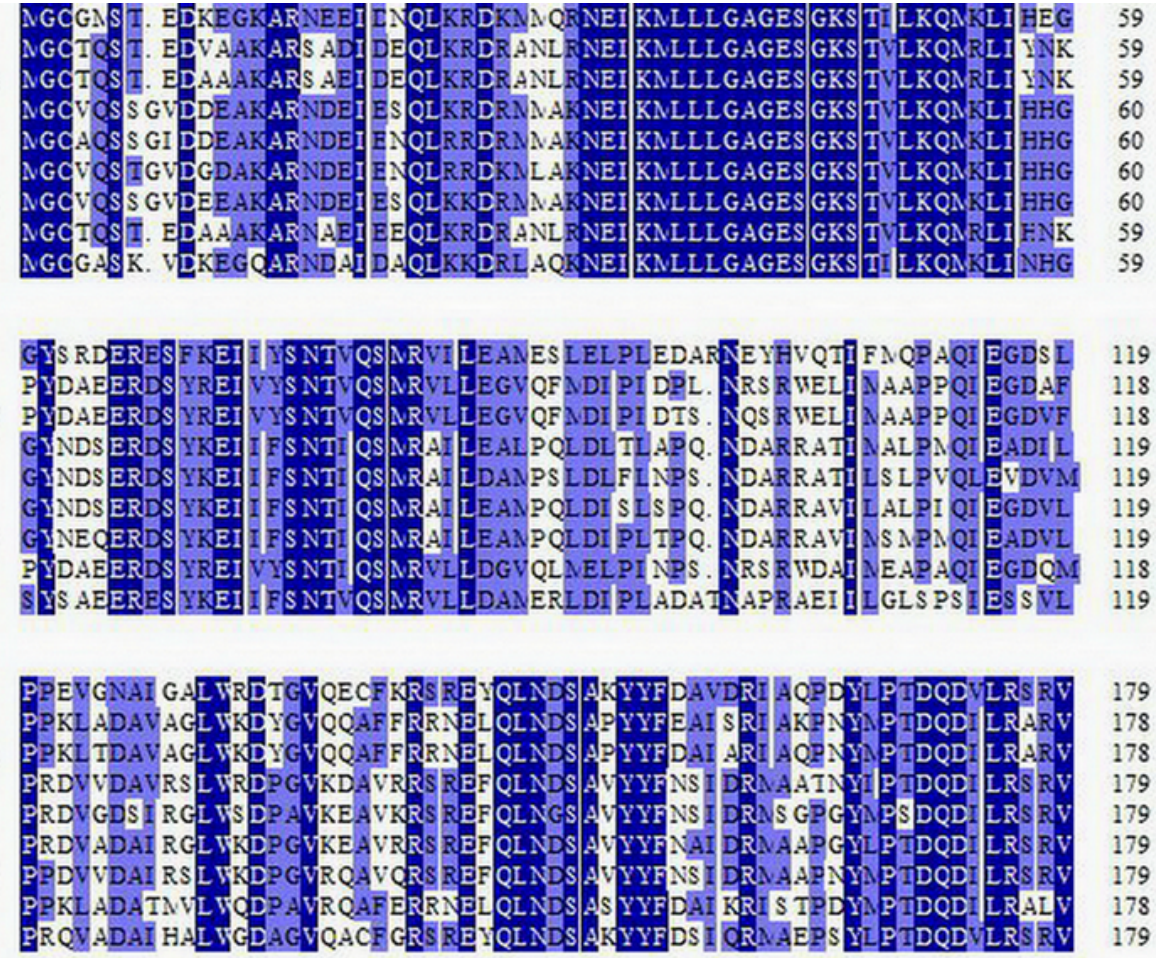

\author{
Aspergillus clavatus \\ Cryptococcus gattii \\ Cryptococcus neoformans \\ Laccaria bicolor \\ Lentinula edodes \\ Pisolithus sp. \\ Schizophyllum commune \\ Tremella fuciformis \\ Ustilago mavidis
}

\author{
Aspergillus clavatus \\ Cryptococcus gattii \\ Cryptococcus neoformans \\ Laccaria bicolor \\ Lentinula edodes \\ Pisolithus sp. \\ Schizophyllum commune \\ Tremella fuciformis \\ Ustilago mavdis
}

Aspergillus clavatus

Cryptococcus gattii Cryptococcus neoformans

Laccaria bicolor

Lentinula edodes

Pisolithus sp.

Schizophyllum commune

Tremella fuciformis

Ustilago mavdis

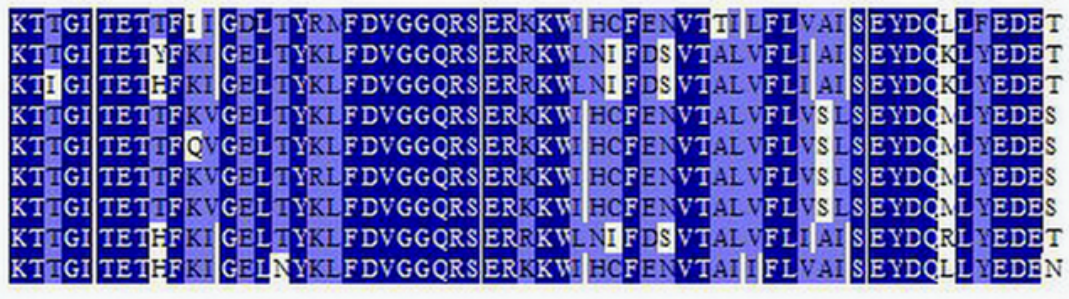

239

238

238

239

239

239

239

238

239

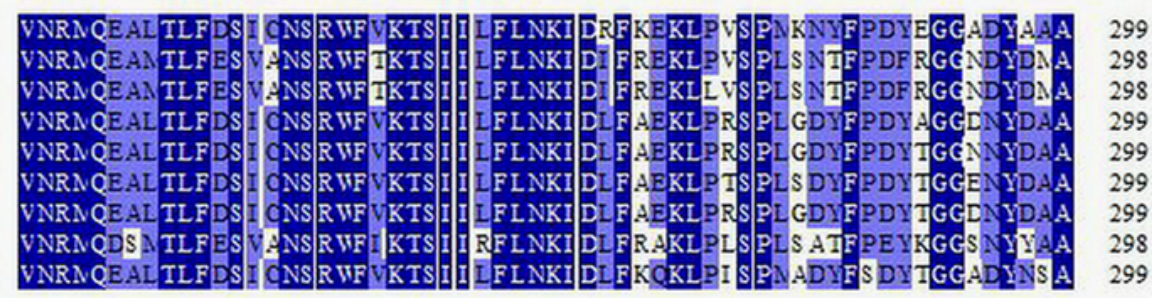

Aspergillus clavatus
Cryptococcus gattii
Cryptococcus neoformans
Laccaria bicolor
Lentinula edodes
Pisolithus sp.
Schizophyllum commune
Tremella fuciformis
Ustilago mavdis

Aspergillus clavatus

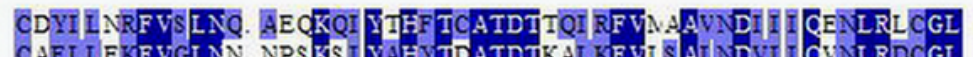

CAFLLEKFVGLNN. NPSKS I YAFYTDATDTKAL KFVI SAI NDVII CVNLRDCGL

CDYLL HRF VS LNQS AATKQI YAHYTCATDTQQI KF VLSAI CDI LL QL LRECGL

CEYLLRRFVSLNQS ATKQVYAHYICATDTQQI KFVLSAI ODI LLQL RLRAGL

CDYLL HRF YINCNAATKQI YAHYCATDTQQI KFVLSAI ODI LLQI HLRECGL

CDYLL HRF VS LNS AATKQI YAHYTCATDTCQI KFVLSAI dDI LLCL HLRECGL

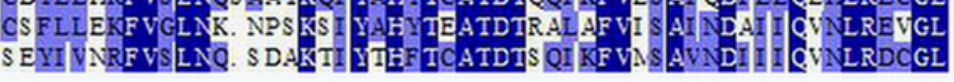

\section{Figure 2}

Multiple amino acid sequence alignment of TrGpa1 with homologues from other fungi: $\mathrm{K}$. heveanensis (OCF36875.1), C. gattii (XP_003191999.1), C. neoformans (XP_566528.1), Pisolithus sp. (AAK15759.1), S. commune (XP_003029155.1), L. bicolor (XP_001888946.1), L. edodes (AAP13579.1), and H. marmoreus (KYQ31721.1). Conserved residues are shown in dark blue boxes, identical resides in light blue boxes, and 
unrelated residues in a white bachground. Dots indicates gaps or the lack of a matching sequence of the protein sequences. Amino acid numbers are shown on the right.

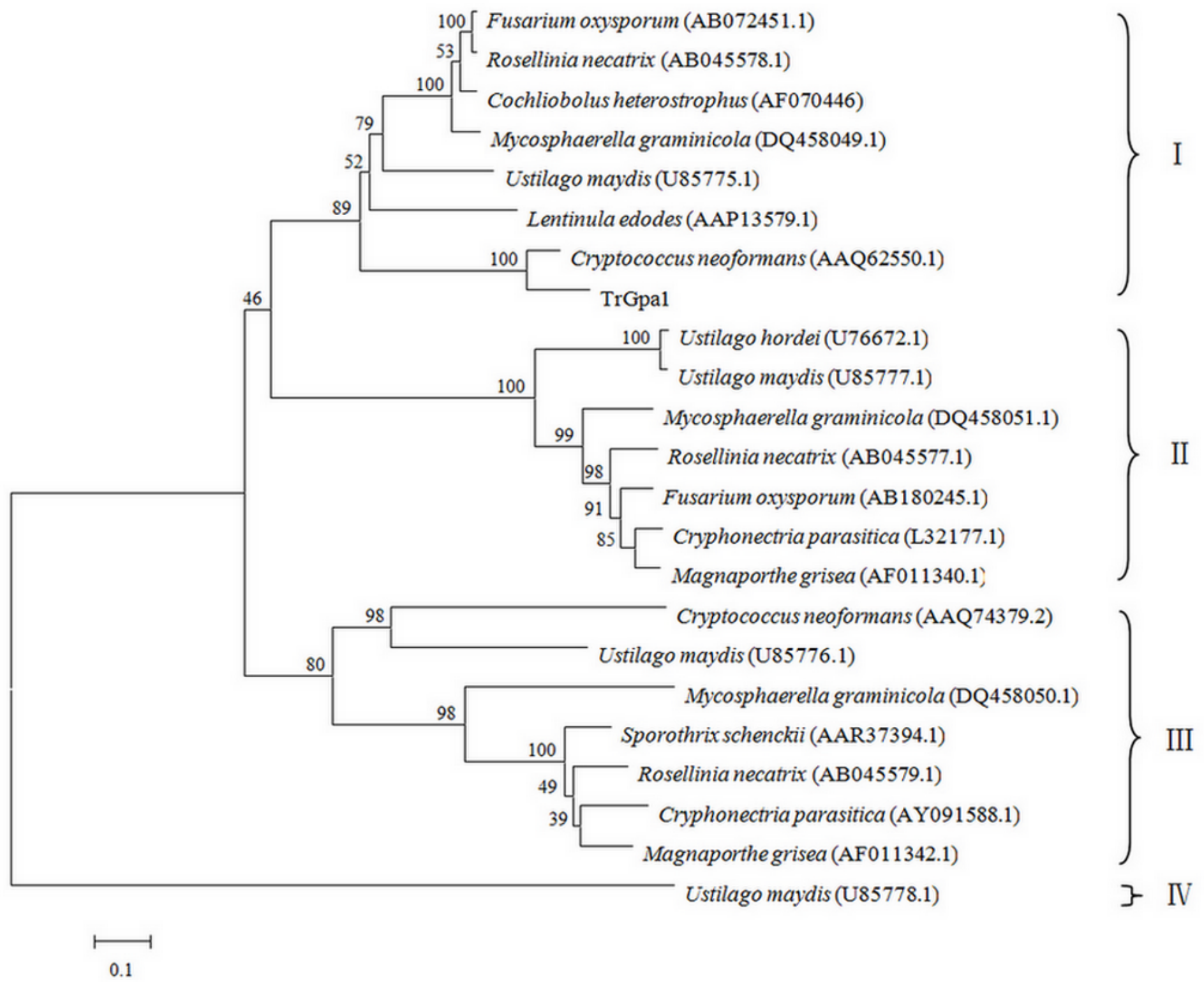

\section{Figure 3}

Phylogenetic tree of TrGpa1 was generated by the neighbor-joining $(\mathrm{NJ})$ method using MEGA 6.0, based on the amino acid sequences of Ga proteins from 22 species. One thousand bootstrap replicates were calculated, and bootstrap values are shown at each node. The scale bar indicates an evolutionary distance of amino acid substitutions per position. 
a

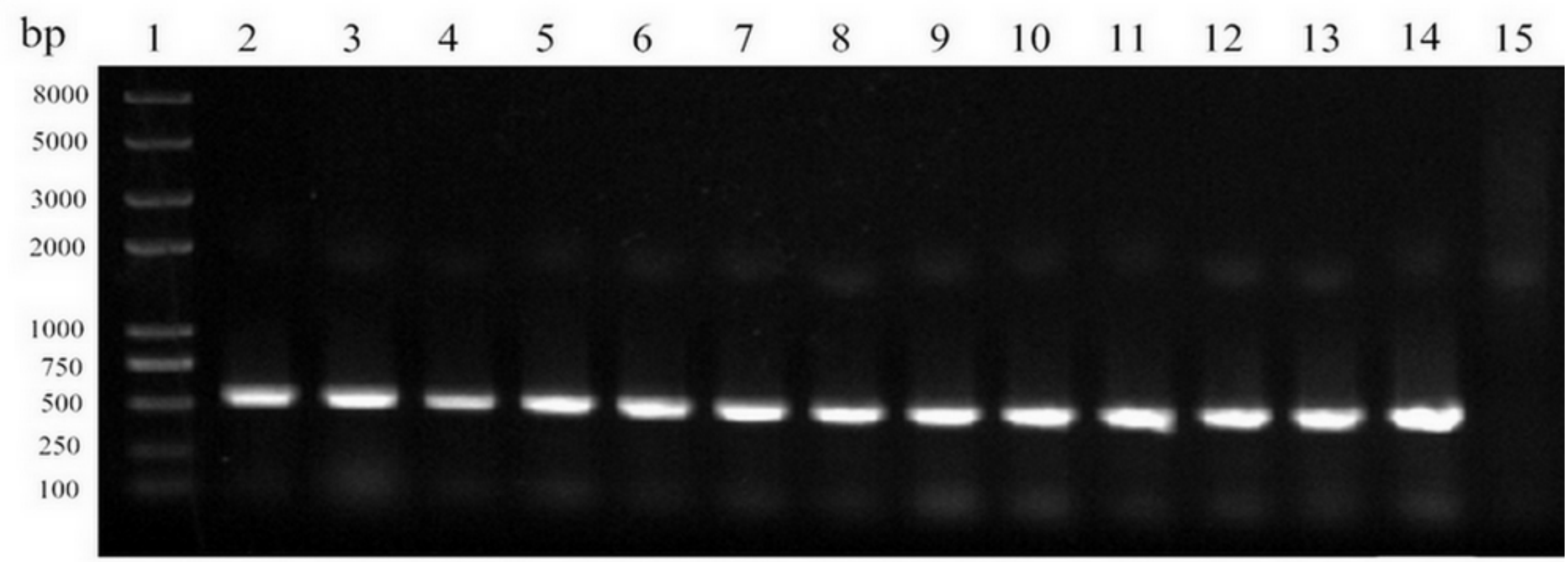

b

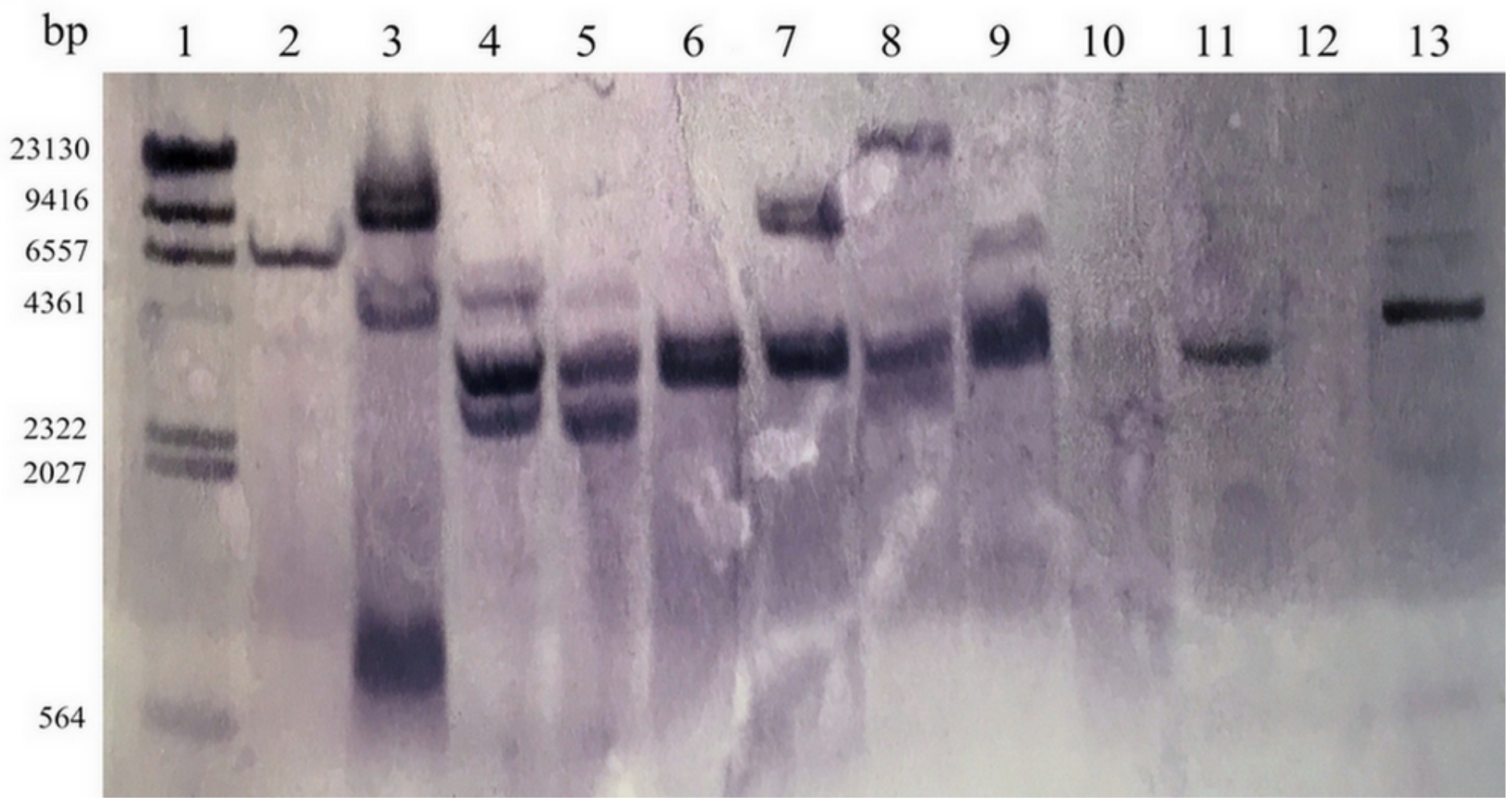

\section{Figure 4}

Stability test of the overexpression and knockdown transformants. a PCR assays of the egfp-hph in the transformants, plasmid pPEH-PH, and Y32. Lane 1, DNA marker. Lane 2-7, PCR products of overexpression transformants. Lane 8-13, PCR products of knockdown transformants. Lane 14, PCR product of pPEH-PH. Lane 15, PCR product of Y32. b Analysis of the integration of hph in T. fuciformis transformants by Southern blot. Genomic DNA digested with Xhol was probed using 500 bp DIG-labeled egfp-hph. Lane 1, DNA marker. Lane 2-6, TrGpa1 overexpression transformants. Lane 7-11, TrGpa1 
knockdown transformants. Lane 12, Y32. Lane 13, pPEH-PH. The molecular weight of DNA marker (bp) is shown on the left.
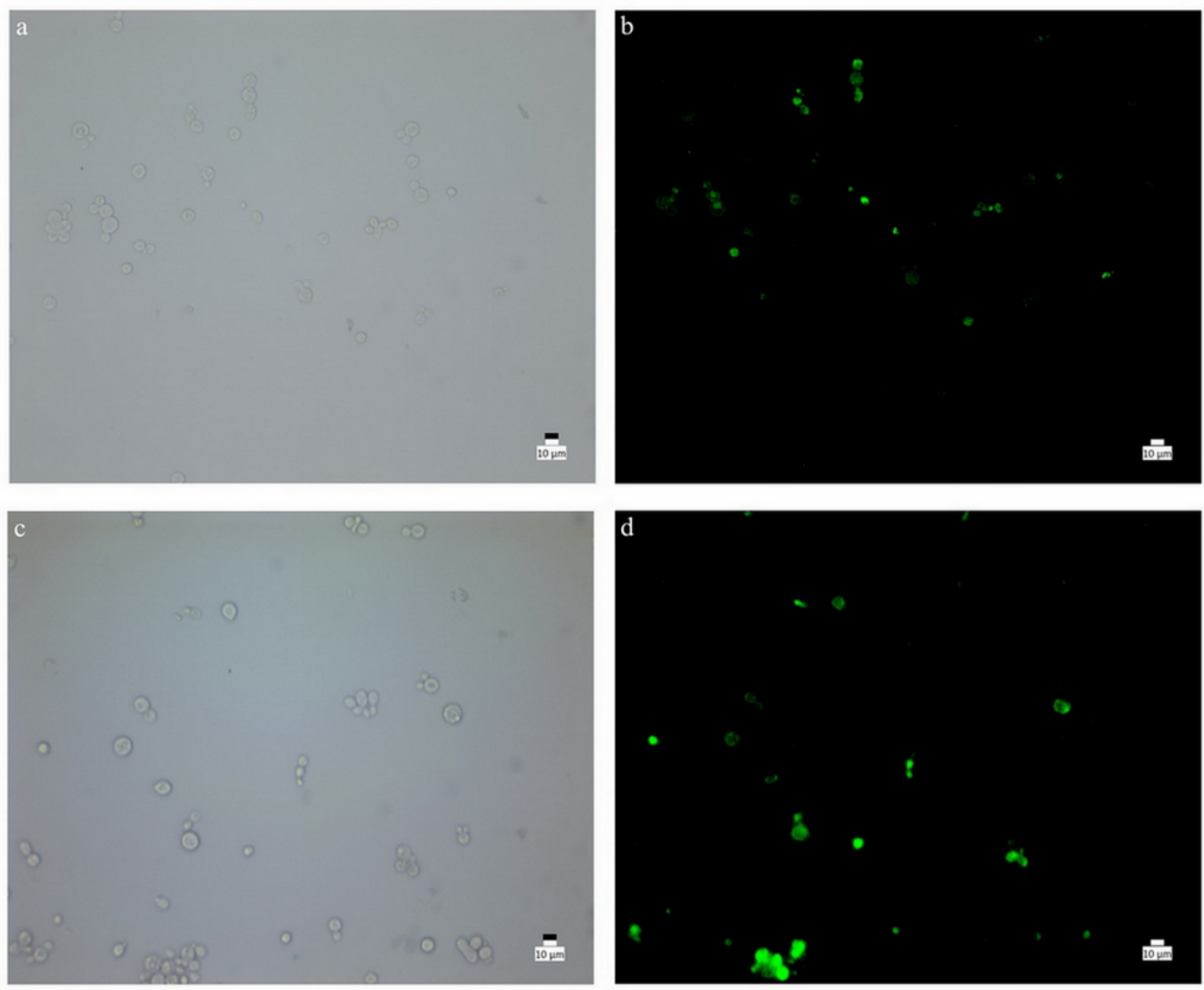

Figure 5

Detection for fluorescence under bright light (a overexpression transformant; c knockdown transformant) and corresponding UV light (b overexpression transformant; $d$ knockdown transformant). Images were taken with $40 \times$ fields of view, bar $=10 \mu \mathrm{m}$. 
a
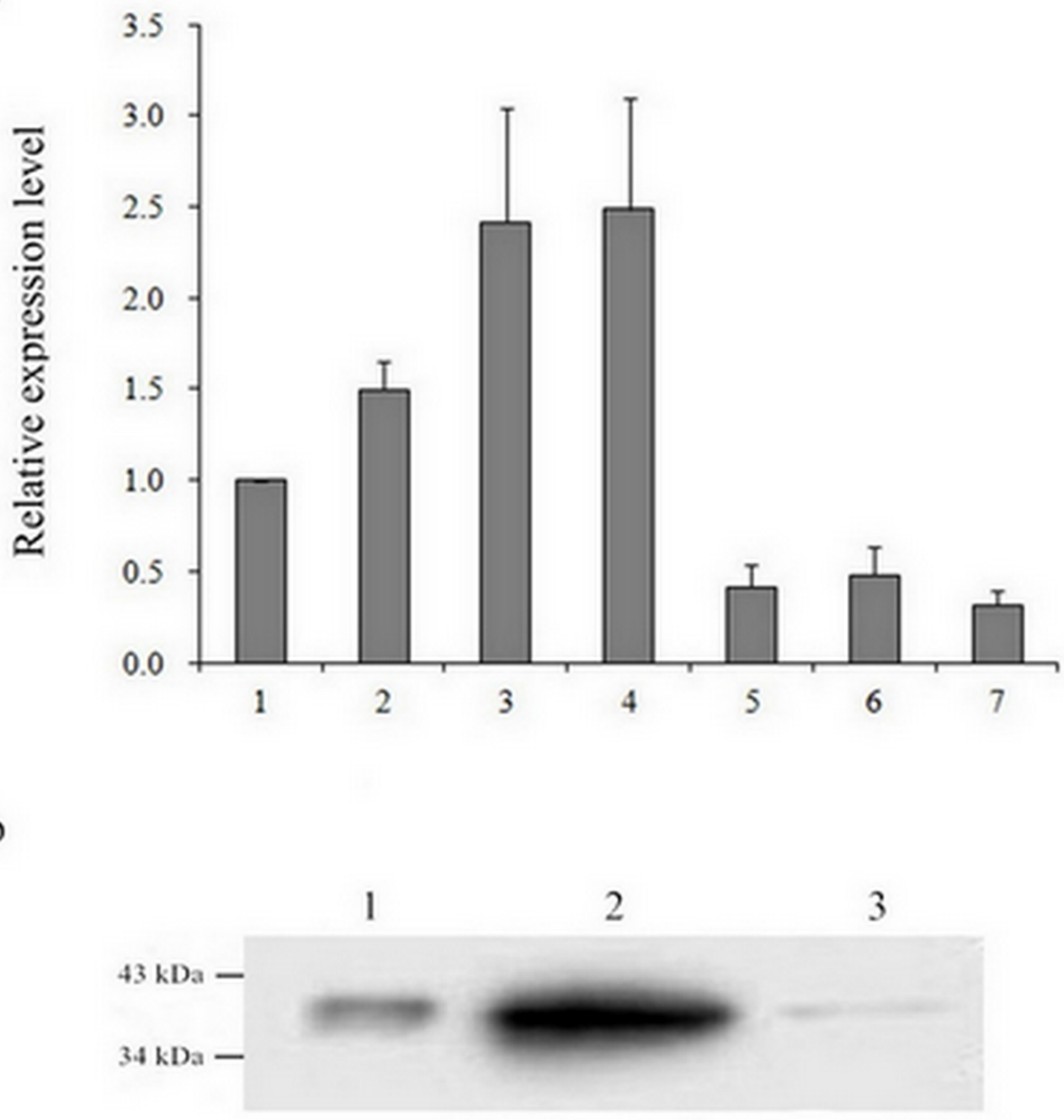

\section{Figure 6}

Gene expression analysis of transformants. a Relative expression levels of TrGpa1 in overexpression (2, $3,4)$ and knockdown transformants $(5,6,7)$ and $Y 32(1)$. Data were presented as mean values of three replicates with the corresponding standard deviations. b Western blot analysis of Y32 (Lane 1), the overexpression transformant (Lane 2), and the knockdown transformant (Lane 3). 

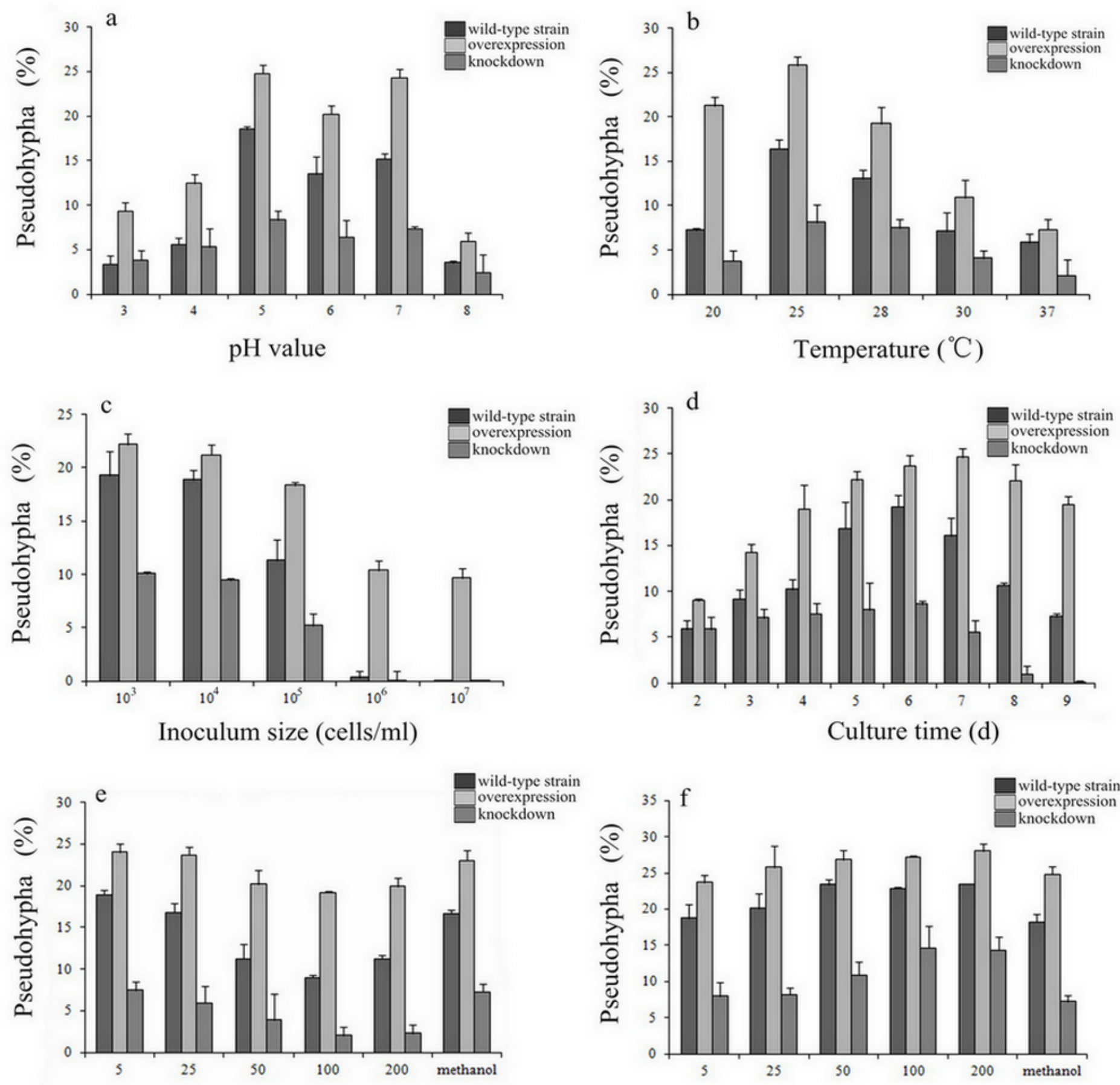

Farnesol concentration $(\mu \mathrm{mol} / \mathrm{l})$

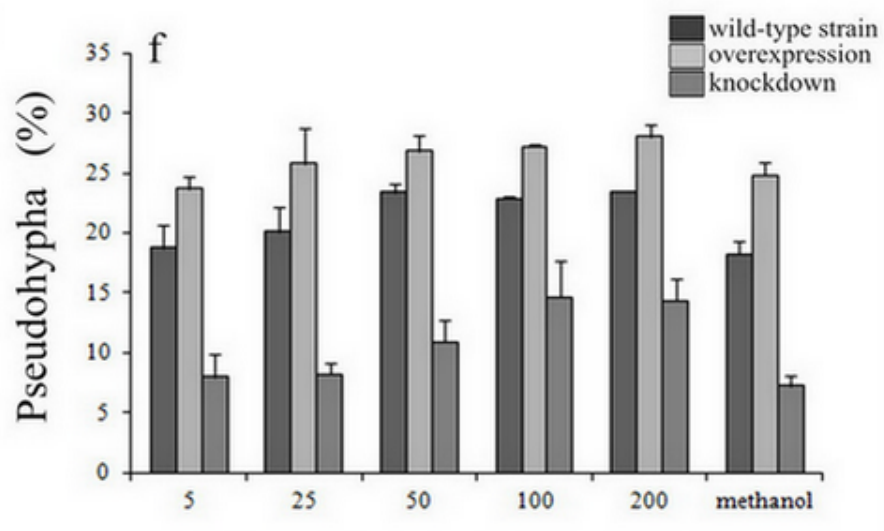

Tyrosol concentration $(\mu \mathrm{mol} / \mathrm{l})$

Figure 7

Effects of $\mathrm{pH}(\mathrm{a})$, temperature (b), culture time (c), inoculum size (d), farnesol (e) and tyrosol (f) on the phenotype of $Y 32$, TrGpa1 overexpression transformant and knockdown transformant. 